\title{
La (dé)nomination du «peuple français » par Emmanuel Macron et Marine Le Pen Débat de l'entre-deux-tours du 3 mai 2017 : une étude lexicale et sémantique
}

\author{
Françoise Sullet-Nylander \& Malin Roitman (Université de \\ Stockholm, Suède)
}

\begin{abstract}
In the proposed article, the authors investigate how the candidates to the French presidential elections, Emmanuel Macron and Marine Le Pen, named their fellow citizens, what types of denominations the two candidates used when referring to the French people during the TV-debate which took place in May 2017. The authors have carried out several discourse analytic studies on the final debate of the French presidential elections (Sullet-Nylander \& Roitman 2010a, 2010b, 2011 and 2016).

This new study deals with the concepts of denomination and/or nomination within a discourse analytic framework based on theoretical models and definitions by Kleiber (2001) and Siblot (2001). The identification of "les paradigmes désignationnels" (Charaudeau \& Maingueneau 2002: 415) enables the collection of relevant semantic and lexical information related to this specific genre and discourse type. The analyses are quantitative and qualitative as well as comparative (between the two candidates).

The following research questions are addressed: How do both candidates refer to French people during their exchanges? Do the differences in the frequency of a particular denomination depend on the political orientation of the candidates? What electoral strategies can be linked to these (de)nominations? The results show several differences between the two candidates, one of which the most salient being Marine Le Pen's use of the lexeme "compatriotes" while Macron prefers "concitoyens".
\end{abstract}

Keywords: Denomination, Nomination, French Political Debate, the French People, Discourse analysis

\section{Introduction}

L'étude qui suit a pour objet le débat de l'entre-deux-tours du 3 mai 2017, entre Marine Le Pen (MLP) et Emmanuel Macron (EM). Ce débat a été décrit par de nombreux observateurs, comme le «plus violent de la Vème République » ou comme un débat qui «a rarement atteint la hauteur et la qualité rhétorique qui caractérise d'habitude en France la parole politique » (Libération du 4 mai 2017) ${ }^{1}$. On peut également citer le chercheur Damon ${ }_{\text {http://www.liberation.fr/politiques/2017/05/04/le-debat-le-pen-macron-vu-par-la-presse-etrangere-le-plus- }}$
violent-de-la-ve-republique_1567224 
Mayaffre $^{2}$, selon lequel la candidate du FN a appliqué une «rhétorique ad hominem ». Malgré ces traits inédits et ces commentaires peu laudatifs sur la dernière édition du débat de l'entredeux-tours, on s'y intéressa ici dans le cadre d'un projet de recherche interdisciplinaire plus vaste sur les discours politiques dans les pays de langue romane ${ }^{3}$.

Les auteures ont déjà mené plusieurs études linguistico-discursives sur les précédents débats de l'entre-deux-tours des élections présidentielles françaises (1974: Giscard d'Estaing/Mitterrand; 1981: Mitterrand/Giscard d'Estaing; 1988: Mitterrand/Chirac; 1995: Chirac/Jospin; 2007: Sarkozy/Royal et 2012: Hollande/Sarkozy). Ces travaux (SulletNylander \& Roitman 2010a, 2010b, 2011 et 2016) ont abordé diverses problématiques, telles que les modes de questionnement, la réfutation, le discours rapporté et, plus récemment, les termes d'adresse dans ce genre "institutionnalisé » qu'est le débat de l'entre-deux-tours, qualifié par Kerbrat-Orecchioni (2017, 16-17) de «genre confrontationnel » et de « compétition impitoyable».

Ce nouveau travail est centré sur les concepts de nomination et de dénomination dans un cadre analytique du discours, basé sur des modèles théoriques et des définitions de Kleiber (2001) et Siblot (2001) en particulier, mais aussi sur les travaux plus récents parus dans les revues Langue Française de 2012 et 2015 consacrées aux problématiques de la dénomination, de la nomination et de la désignation. Nous y reviendrons dans la présentation de notre cadre théorique (section 2). Il s'agit ici d'une étude quantitative et qualitative sur la façon dont les deux candidats font référence au «peuple français » au cours de leurs échanges. Les objectifs principaux de l'étude sont ainsi, de relever les formes nominales utilisées par EM et MLP pour renvoyer explicitement au «peuple français » au cours du débat du 3 mai 2017 et de comparer l'emploi de ces formes entre les deux candidats-

Les questions de recherche suivantes sont abordées : comment les deux candidats se réfèrent-ils au peuple français lors de leurs échanges ? Les éventuelles différences de fréquence dans l'emploi de telle ou telle (dé)nomination dépendent-elles de l'orientation politique du/de la candidate ? Ou bien s'agit-il pour chaque candidat de renforcer son ethos préalable en employant telle forme nominale plutôt que telle autre ? Enfin, quelles stratégies électorales peuvent être liées à ces (dé)nominations ?

\section{De la dénomination à la nomination en passant par la désignation ?}

Comme le signale Frath $(2015,33)$, le débat sur la dénomination reste tout à fait d'actualité si on en juge par les deux numéros de Langue Française (174 et 188) parus en 2012 et 2015. Selon ce chercheur, la réflexion linguistique sur la question de la dénomination remonte aux années 80 avec, en particulier, les travaux de Kleiber (1981 et 1984). Dans un article de 2001, paru dans les Cahiers de praxématique, ce dernier réexamine son approche de la dénomination et met en avant ce qu'il nomme le «paradoxe sémantique de la dénomination ». Kleiber $(2001,22)$ prend comme point de départ la définition suivante :

\footnotetext{
${ }^{2}$ Ce chercheur s'exprime au lendemain du débat dans le journal Nice Matin : http://www.nicematin.com/politique/desarconnant-ce-nicois-specialiste-du-discours-politique-livre-son-analysedu-debat-macron-le-pen-134340

${ }^{3}$ La présente étude se place dans le cadre du projet de recherche interdisciplinaire ROMPOL, conduit par des linguistes et des chercheurs en sciences sociales, au département d'études romanes et classiques de l'Université de Stockholm. Le projet cherche à analyser les discours politiques de divers pays de langues romanes, aussi bien en Europe qu'en Amérique Latine.
} 
[L]a dénomination doit être envisagée comme une relation entre une expression linguistique $X$ et un ou des éléments ou choses de la réalité $x$. On appellera aussi dénomination l'expression linguistique $X$ en question. La dénomination s'inscrit donc dans la problématique sémiotique du signe qui renvoie à autre chose qu'à lui-même : il n'y a signe que s'il y a signe de quelque chose.

Il faut également distinguer, selon lui, la dénomination de la désignation, en tant que celle-là correspond à une "habitude associative », tandis que celle-ci peut désigner quelque chose par une expression, sans que ce quelque chose ait été désigné auparavant. Kleiber $(2001,23)$ parle alors d' « association référentielle durable et non contingente de $\mathrm{x}$ »

Selon Siblot $(2001,189-214)$ dans la même revue, la dénomination relèverait d'une approche statique établie au plan de la langue, tandis que la nomination consisterait en un acte de parole contextualisé et serait donc assigné au champ du discours. Dans cette approche, les stratégies discursives des locuteurs et les contextes situationnel et communicationnel occupent une place importante. Ce chercheur affirme ainsi que les termes sont des «marqueurs de positions » et des outils pour produire du sens et non de simples «étiquettes ». À travers son positionnement de linguiste du discours ou au sein de ce qu'il nomme une «sémantique réaliste ${ }^{4}$, l'auteur revendique ainsi le terme de «nomination» pour tout acte de catégorisation nominale et rapproche la problématique de la nomination à celle du dialogisme, tel que décrit par Bakhtine $(1929,67)$ : «Chaque mot, nous le savons, se présente comme une arène en réduction où s'entrecroisent et luttent les accents sociaux à orientation contradictoire. Le mot s'avère, dans la bouche de l'individu, le produit de l'interaction vivante des forces sociales ».

Gérard Petit (2002), quant à lui, considère que la notion de dénomination n'a pas donné lieu à une conceptualisation particulière, contrairement à celle de désignation, qui est à l'origine de ce que l'analyse du discours (désormais AD) appelle «paradigmes désignationnels $»^{5}$. Le repérage de ces paradigmes permet de recueillir des informations pertinentes non seulement du point de vue de la sémantique lexicale, mais aussi dans la perspective de caractérisation d'un discours (Charaudeau \& Maingueneau (dir) 2002, 415).

Les récents travaux traitant de ces questions articulent ces trois notions et envisagent la nomination comme une catégorie discursive. Dans le numéro de Langue Française (2015), on constate la «grande hétérogénéité » dans les choix conceptuels et dans la terminologie, comme en témoigne l'inventaire fait par Moirand et rapporté dans Longhi $(2015,6)$ : référence, stratégies de désignation, mots, dénomination, nommer, catégorisation, référenciation, paradigmes désignationnels, nomination, désignants.

Frath $(2015,34)$ établit, lui aussi, une opposition nette entre d'un côté la dénomination (donnée par la langue) et de l'autre, la désignation (« référence discursive individuelle ») et la nomination («tentative pour donner un nom à un nouvel objet de notre expérience collective »). Il départit ainsi ces deux dernières notions (les italiques sont de l'auteur) : « Si les dénominations sont lexicalisées et jouissent ainsi d'une existence ontologique dans la

\footnotetext{
${ }^{4}$ C'est également ce terme de « linguistique réaliste » qu'utilise Laura Calabrese $(2012,29)$ dans son article sur l'acte de nommer dans le discours médiatique : «Pour l'Analyse du discours française (ADF), le langage s'articule inévitablement au réel, dans la mesure où il dit le monde, le façonne ou contraint les représentations que nous en avons. L'ADF naît en effet comme une « linguistique réaliste », opposé à la réification du signe proposée par le paradigme structuraliste. »

5 Nous référons ici à l'article de Gérard Petit dans le Dictionnaire d'analyse du discours dirigé par Charaudeau \& Maingueneau $(2002,164)$.
} 
langue, ce n'est pas le cas des désignations ni des nominations ». (Frath 2015, 36). Enfin, citons Longhi $(2015,8)$, qui résume ainsi la problématique des domaines dont relève le traitement du couple dénomination $v s$ nomination :

Si la recherche sur la dénomination relève davantage de la lexicologie ou de la sémantique, les travaux sur la nomination ont généralement été produits dans le champ de l'AD. Or, si l'on donne comme ambition à l'AD de proposer une sémantique du discours qui permette l'analyse rigoureuse du sens en discours, il faut pouvoir proposer une prise en compte du sens qui puisse opérer dans les discours eux-mêmes, et non pas seulement sur des énoncés isolés.

C'est ainsi, dans la perspective de l'AD ou «linguistique réaliste », que nous aborderons à présent l'analyse de notre corpus, avec l'objectif de comparer les nominations $\mathrm{du}$ «peuple français » utilisées par les deux candidats au cours du même débat, ceci afin de cerner ce qui révèle le «positionnement de l'énonciateur » (Charaudeau et Maingueneau (dir) 2002, 416).

\section{Méthodologie et repérage des formes nominales}

Après avoir transcrit intégralement le débat du 3 mai $2017^{6}$, nous avons procédé au repérage de noms/formes nominales référant explicitement au "peuple français ». Dans un premier temps, nous avons prélevé un vaste nombre de nominations renvoyant au peuple au sens large, en particulier celles relevant de catégories socio-économiques et de catégories en rapport à l'appartenance religieuse, à la famille, à l'entreprise ou à l'immigration. Nous y reviendrons brièvement en 4.1. Pour l'analyse qualitative (4.2-4.4), nous nous concentrerons sur les nominations renvoyant au concept de «peuple français » dans son acception plus «étroite », c'est-à-dire dans le contexte électoral, où il s'agit pour chaque candidat de s'adresser ou de référer au peuple dans ses fonctions civiques. Nous nommons cette catégorie «identité nationale et civique» (voir 4.2-4.3). En effet, notre intérêt porte plus particulièrement sur la représentation de la nationalité dans un sens culturel et historique, ainsi que sur l'image que les représentants des partis politiques en lice se font de leur électorat. Nous nous intéressons également à la question de l'ethos présidentiable qui, selon nous, est liée au choix d'inclure ou non certains groupes dans son discours et à la façon dont les candidats choisissent de nommer leurs potentiels électeurs. Notre analyse portera ainsi aussi bien sur la quantité des données (nombre de nominations) ainsi que sur la qualité des données (types de nominations).

\section{Analyse}

Nous présenterons d'abord brièvement, en 4.1, les résultats quantitatifs englobant toutes les nominations du débat référant au peuple. En 4.2 et 4.3 , nous traiterons de nominations relevant de la catégorie « identité nationale et civique », en mettant l'accent sur

\footnotetext{
${ }^{6} \mathrm{Au}$ moment de la préparation de notre communication pour le Congrès des Romanistes Scandinaves de Bergen (août 2017), dont est issu cet article, il n'existait, à notre connaissance, aucune transcription du débat de l'entredeux tours du 3 mai 2017. Nous avons donc fait nous-même cette transcription. Il s'agit d'une transcription orthographique à partir du débat diffusé sur https://www.francetvinfo.fr/elections/presidentielle/directpresidentielle-le-debat-emmanuel-macron-marine-le-pen-du-2eme-tour-a-suivre-en-live-sur-france-2-et$\underline{\text { tf1_2173168.html }}$
} 
les effets rhétorico-discursifs véhiculés par l'usage de ces nominations. En effet, le fait de choisir telle ou telle nomination peut révéler la nature de l'ethos présidentiel des candidats, compte tenu de la situation politique en France et en Europe aujourd'hui, où les questions d'immigration et de citoyenneté sont centrales. Cette hypothèse est aussi liée au fait que l'un des candidats représente un parti d'extrême droite, dont la politique est centrée sur la protection du territoire, de l'économie et du patrimoine national. En 4.4, nous mettrons nos résultats concernant la catégorie «identité nationale » en regard des autres catégories, afin de mettre la totalité des nominations en relation avec le contexte. Ainsi nous espérons pouvoir encore discerner des tendances globales dans les discours de chacun des candidats.

\subsection{Résultats quantitatifs toutes catégories confondues et premières analyses}

Au total, les deux candidats désignent les citoyens français 466 fois : Macron 246 fois et Le Pen 220 fois. Concernant la répartition, la plupart des nominations proviennent de la catégorie que nous nommons identité nationale (ex. «(les) concitoyens », «(les) concitoyennes », «(les) compatriotes », « les Français », « les Françaises » «(le) peuple », «(le) peuple français », etc.). Ils y réfèrent 177 fois au total : 89 fois pour EM et 88 fois pour MLP. Les nominations venant à la deuxième place sont celles qui renvoient aux personnes exerçant un métier (catégorie "profession") : "commerçants", "agriculteurs", etc. Ces nominations sont au nombre de 145 et c'est EM qui emploie le plus de nominations renvoyant à la population active. Les nominations sous la catégorie «entreprises » réfèrent aux entrepreneurs français sous différentes formes : «TPE-PME », " grandes sociétés », etc. Macron se sert 51 fois et Le Pen 42 fois de nominations référant directement aux personnes dirigeant une entreprise, grande ou petite. La candidate du FN utilise plus souvent des nominations renvoyant à la catégorie «non-actifs », avec des formes comme « retraités » et « chômeurs » (6 vs 1 fois et 6 vs 2 fois). ${ }^{7}$.

Quant aux types et à la nature sémantique des nominations choisies, constatons que pour la catégorie «identité nationale et civique », ces formes représentent une assez grande variation sémantique et que les choix opérés par chaque candidat varient également (voir 4.24.3). Constatons enfin que dans la catégorie socio-économique ne figurent que des métiers traditionnellement masculins et pratiques comme « agriculteur», «commerçant» et « éleveur» et des professions relatives à l'ordre et à la sécurité, comme "gendarmes », «policiers », «juges », «douaniers », «forces de l'ordre », etc. Ni l'un ni l'autre ne fait mention de métiers traditionnellement féminins comme institutrices, infirmières, aidessoignantes, etc. EM est cependant plus neutre en privilégiant la forme «salariés », tandis que Le Pen emploie «travailleurs» dans des contextes concernant les conditions de travail, généralement mauvaises, des travailleurs. Regardons maintenant de plus près la catégorie «identité nationale et civique ». Notre objectif est de discerner dans quelle mesure ces nominations du peuple français attribueraient un éthos spécifique à chacun des deux candidats.

\footnotetext{
${ }^{7}$ Un petit nombre de nominations sont classées dans la catégorie "identité civique". Il s'agit des formes "les enfants", "les jeunes", "les ménages", "la famille", etc. EM s'en sert 23 fois des nominations de cette catégorie et MLP s'en sert 13 fois.
} 


\subsection{Identité nationale et civique I: « concitoyens »/ « concitoyennes »vs « compatriotes»}

Les deux formes «concitoyen » et «compatriote » sont définies ainsi dans le Grand Robert (1990) :

a. Compatriote: personne originaire du même pays; personne originaire de la même province, de la même région qu'une autre (à l'intérieur du même pays) > concitoyen ;

b. Concitoyen : citoyen du même État, d'une même ville (qu'un autre) > compatriote

Pourtant dans le contexte politique où elles apparaissent, ces formes véhiculent des valeurs différentes, d'autant que les morphèmes dont elles se composent - «citoyen » et «patriote »- renvoient à des rapports différents vis-à-vis du même pays/État, le premier plus neutre et plus juridique, tandis que le second renvoie à l'amour du pays, de la patrie. C'est seulement dans un sens vieilli que les deux formes sont synonymes (Le Grand Robert 1995) : «Patriote : vx Compatriote (encore in Rousseau, Lettres, 2 août 1766) - Citoyen.

Dans le corpus, les occurrences se distribuent ainsi : EM produit 30 fois la forme «concitoyens» et 2 fois «citoyens », tandis que MLP produit, elle, 6 fois la forme « compatriotes », 1 fois la forme « citoyens », dans le syntagme « «d'honnêtes citoyens », et 1 fois la forme «patriotes ». Dans son discours d'introduction, alors qu'elle répond à la question de la journaliste («Une première question, après des mois de campagne, à quatre jours du second tour et à 10 jours de votre investiture si vous êtes élu(e), quel est votre état d'esprit, Marine Le Pen ? »), MLP s'exprime ainsi :

(1) Je suis la candidate de la nation qui protège, qui protège nos emplois, qui protège la sécurité de nos compatriotes qui protège nos frontières, qui nous protège face à la concurrence internationale déloyale et face à la montée en puissance du fondamentalisme islamiste.

Elle se présente ainsi, à plusieurs reprises, comme la candidate qui va assurer la "préférence nationale » aussi bien en ce qui concerne l'emploi (2) que la santé (3) et la sécurité (4) :

(2) Les TPE-PME qui réclamaient qu'on aille leur alléger leurs difficultés, leur donner de l'oxygène, vous avez fait comme s'ils n'existaient pas, parce que vous avez refusé de supprimer la directive détachement des travailleurs et qu'aujourd'hui ce sont 300 à 500000 emplois qui en réalité ne sont pas accessibles à nos compatriotes français parce qu'ils sont remplis par des travailleurs détachés.

(3) Hein, puisqu'un Français sur trois ne se soigne plus correctement aujourd'hui... parce qu'encore une fois, on ne réserve pas la solidarité nationale à nos compatriotes.

(4) La sécurité et le terrorisme est une problématique absolument majeure. Totalement absente de votre projet. Totalement absente. Aucune proposition qui tienne la route. C'est-à-dire que la sécurité physique de nos compatriotes et la lutte contre le terrorisme et le fondamentalisme islamiste, vous ne voulez pas le mener et vous ne voulez pas le mener monsieur Macron, moi je sais pourquoi.

EM quant à lui n'utilise aucune occurrence de «compatriotes ». Il utilise de manière conséquente, tout le long du débat, la forme nominale «concitoyens » (30 fois) pour référer 
aux Françaises et aux Français. Dans son introduction, répondant à la même question que MLP mentionnée plus haut :

(5) La question qui est posée aujourd'hui à nos concitoyens, c'est de savoir s'ils veulent l'esprit de défaite que vous portez, parce ce que vous portez, c'est l'esprit de défaite. C'est d'expliquer à nos concitoyens, c'est trop dur la mondialisation pour nous, c'est trop dur l'Europe, donc on va se replier, on va fermer les frontières, on va sortir de l'Euro, sortir de l'Europe parce que les autres y arrivent mais pas nous.

Mais aussi dans sa conclusion, où il s'adresse directement $\left(2^{\text {ème }}\right.$ occurrence) aux Français et Françaises :

(6) Un monde où nous pouvons nous protéger, parce que nous aurons une politique volontariste, parce que nous aurons formé nos concitoyens, parce que nous aurons su répondre à ces défis. Je refuse l'esprit de défaite et de haine que porte le Front National. Je veux un esprit de conquête avec des vraies réformes et des vrais changements. Nous le ferons ensemble, mes chers concitoyens.

À travers l'étude de ces premières nominations et de leur contexte, on constate une répartition nette entre les deux candidats, avec d'un côté la représentation du peuple comme un ensemble de personnes responsables et conquérantes (chez EM) et de l'autre, celle de personnes attachées aux valeurs traditionnelles de la patrie (chez MLP).

\subsection{Identité nationale et civique II : «Les Français »/«Les Françaises », "Le peuple » / «Le peuple français », "La France » et «Les gens»}

En ce qui concerne les formes «Les Français »/ « Les Françaises» «Peuple»/«Peuple français », «La France », « les gens », les différences quantitatives ne sont pas énormes entre les deux candidats mais, si l'on étudie les types de nominations, on constate qu'il y a une tendance, de la part de MLP, à se servir davantage de syntagmes nominaux renvoyant à la nationalité française qu'EM. Ce sont des nominations en principe neutres, puisqu'elles ne témoignent que d'une appartenance géographique, juridique et culturelle. Voyons à présent plus en détails dans quels contextes ces formes apparaissent et avec quels effets.

\subsection{1 «Les Français »/« Les Françaises »}

Les contextes de ces nominations sont révélateurs des deux attitudes opposées : là où MLP voit les «Français » comme souffrants, frustrés, soumis et victimes des circonstances, EM considère les «Français » et les «Françaises » comme des individus travailleurs, actifs, intelligents et capables. Dans le discours de MLP, la plupart des emplois (14 sur 20) de « les Français » se font dans des contextes où elle les associe à un manque de pouvoir et à une position de victimes des circonstances. MLP se présente également comme la sauveuse de ces «naufragés ». En voici quelques exemples :

(7) MLP : «Vous ne le ferez pas parce que vous êtes soumis à eux Monsieur Macron, parce qu'ils vous tiennent. Voilà c'est aussi simple que cela. C'est malheureux à dire. C'est terrifiant, car cela peut avoir des conséquences extrêmement lourdes sur les Français. » 
(8) MLP : «Si ce n'est pas moi qui protège les Français - je protègerai l'épargne des Français quel qu'en soit le prix et quelle que soit la bagarre que je dois mener avec l'Union Européenne - alors les Français se feront ponctionner leur épargne. Le grand danger aujourd'hui, Monsieur Macron, c'est la soumission à l’Union Européenne...

En ce qui concerne EM, la plupart (8 sur 13) des dénominations « les Français »sont utilisées dans un contexte narratif où le candidat du mouvement «En Marche » parle surtout des capacités des «Français » à résoudre des problèmes. Il est également intéressant de constater que c'est uniquement EM qui utilise la forme féminine «Françaises »:

(9) EM : «... Je vous dis juste que moi, je prends les Françaises et les Français pour des adultes. Je ne leur mens pas.

(10) EM : «Les Français ne sont pas aussi bêtes que vous le pensez. »

(11) EM : Non, non, ce qui est extraordinaire, c'est que vous ne répondez en fait jamais aux questions. Vous parlez toujours du passé et des autres, mais c'est très bien, les Français comprendront que vous n'avez rien à proposer. »

\subsection{2 «le peuple »/« le peuple français »}

D'un point de vue quantitatif, c'est MLP qui emploie le plus « le peuple » et «peuple français ». Quand elle parle du « peuple », elle semble vouloir évoquer le concept d'un peuple homogène partageant les mêmes valeurs socio-culturelles, issu d'une même et unique sphère culturelle, comme dans les exemples (12 et 13). Cependant, elle attribue aussi au «peuple » un rôle de victime, ayant besoin de protection (14 et 15):

(12) MLP : « «Bon eh bien moi face à cela, je suis la candidate du peuple, je suis la candidate de la France telle que nous l'aimons, de sa culture, de sa civilisation, de son unité... »

(13) MLP : «Je crois que la France est une nation avec une culture, avec un peuple, avec une espérance qui est l'espérance de pouvoir continuer dans des conditions qui soient des conditions respectueuses; commercer avec les autres nations du monde, rétablir une diplomatie. »

(14) MLP: «Donc, le projet qui est mené par les puissances financières qui vous soutiennent... et qui visent à laisser le système tel qu'il est... parce que c'est à leur bénéfice en réalité. L'Euro, c'est la monnaie des banquiers, mais ce n'est pas la monnaie du peuple.»

(15) MLP : « Si nous ne retrouvons pas notre place diplomatique avec cette voix, qui est la voix de l'indépendance, qui est la voix de la souveraineté, qui est la voix encore une fois de la défense des peuples. »

Quand EM parle du « peuple » il semble inclure tous les Français, sans exception :

(16) EM : «Le peuple français fera son choix ».

(17) EM : «Non il faut une réforme qui a des contraintes constitutionnelles, comme vous le savez, et je n'exclus pas d'aller devant le peuple s'il y avait un blocage des assemblées. 
Ainsi, c'est de sa part une désignation plutôt neutre pour parler des citoyens et des électeurs français.

\subsection{3 «La France»}

La forme «La France » est quantitativement la plus importante parmi celles renvoyant à l'identité nationale française, et c'est à MLP que revient le plus haut score. Quand la candidate du FN parle de «la France », elle l'associe à un peuple victime, mécontent, sans pouvoir, et exposé à des dangers (ex.18, 19 et 20). Mais «la France » représente aussi chez MLP (4 sur 7 fois) un groupe homogène, avec une culture et une voix unique dans le monde, un pays modèle dont les Français devraient être fiers : «le monde attend la France » (22). Il y a des effets de personnification autours de cette désignation : «La France a été jetée dans le chaos » (19) et « la France a perdu cette voix particulière » (22):

(18) MLP: Vous dites, si on ne fait pas une Europe fédérale totalement, avec un gouvernement de la Zone européenne, c'est-à-dire avec la perte de notre souveraineté totale, avec l'intégralité des clefs de la France données dans tous les domaines de l'Union Européenne...»

(19) MLP : La France a été jetée dans le chaos par vos amis politiques et par ceux qui les ont précédés, c'est-à-dire par tous ceux qui vous soutiennent dans cette campagne, Monsieur Macron.

(20) MLP : C'est la France que vous voulez, vous. C'est-à-dire une France qui est ouverte à tous les vents. »

(21) MLP : je suis la candidate de la France telle que nous l'aimons, de sa culture, de sa civilisation, de son unité...»

(22) MLP: Non, la France sera respectée si elle est la France. Si elle redevient véritablement la France avec cette voix particulière qu'elle avait dans le monde. Parce que le monde attend la France. Et la France a perdu cette voix particulière parce qu'elle s'est soumise précisément à l'Allemagne.

EM parle, dans la majorité des exemples, de «La France » comme d'une force économique dans le futur (ex. 23 et 24). Mais «La France » apparaît aussi dans son récit comme «un pays dans le monde», une nation ouverte à des réformes, à des relations économiques et culturelles avec d'autres pays et surtout l'Europe (ex. 25, 26 et 27).

(23) EM : «Donc, le projet qui est le mien, c'est d'avoir une France qui est compétitive. »

(24) EM : «Et de construire une politique européenne de construire une politique européenne qui sera forte et dans laquelle nous défendrons les intérêts de la France. »

(25) EM : «Je dis juste que la France n'est pas un pays fermé, c'est un pays qui est dans l'Europe et dans le monde. La question ça n'est pas l'esprit de défaite que vous voulez. »

(26) EM : «Moi la ligne que je veux avoir pour la France, c'est une ligne que je qualifierais de gaullo-mitterrandienne. C'est l'indépendance de la France. » 
(27) EM : Mais vous avez raison. La France, c'est bien autre chose. C'est une civilisation ouverte avec des principes généreux. Tout l'inverse de ce que vous portez.

Les récits encadrant ces désignations dans le discours de chaque candidat permettent de discerner nettement ces tendances.

\subsection{4 «les gens»}

Cette dénomination apparaît dans des contextes plutôt neutres chez les deux candidats, même si la même tendance que pour les autres nominations se dessine. C'est surtout EM qui emploie «gens» pour désigner l'identité nationale française en mettant l'accent sur leur capacité et intelligence :

(28) EM : «mais les gens ne sont pas bêtes »

MLP de son côté maintient son aptitude à associer le peuple à une communauté soumise et exposée à l'arrogance et au mépris de la société mais aussi de EM et de son parti :

(29) MLP : «Il faut aussi valoriser considérablement les filières professionnelles, parce que là encore un certain nombre de bobos ont considéré qu'il fallait que tout le monde ait bac +12 et que tous ceux qui travaillaient avec leurs mains étaient des gens qui ne méritaient même pas d'être considérés ...

(30) MLP : «Vous voulez dire les ouvrières de Gad que vous avez traitées d'illettrées. Vous voulez dire les chômeurs que vous avez envoyés acheter un costard. Vous voulez dire les gens du Nord que vous avez traités d'alcooliques...

\subsection{Tendances globales du corpus et contextualisation}

Nous avons également analysé les catégories socio-économiques discutées en 4.1, dans leur contexte. Concentrons-nous ici uniquement sur les tendances de victimisation (MLP) et celle de valorisation (EM) du peuple et constatons que les résultats de l'analyse de ces formes confirment ceux de l'analyse de l'identité nationale présentés en 4.2 et 4.3. Pour cette catégorie, on observe que sur les 88 nominations de MLP, référant à l'identité nationale, 43 sont utilisées dans un contexte où le peuple est représenté comme «victime » ou « mécontent ». Dans la même catégorie, sur les 89 nominations référant à l'identité nationale d'EM, 29 sont présentées dans un contexte qualifiant le peuple de «capable ». En ce qui concerne l'analyse discursive des catégories socio-économiques, nous ne pouvons, faute d'espace, que donner un bref aperçu des résultats. Force est de constater que, indépendamment des nominations choisies par les deux candidats - «les Français» (catégorie «identité nationale et civiques»), les «travailleurs» (catégorie socio-économique «professions »), les «TPE-PME » (catégorie socio-économique «entreprises » -, les tendances sont nettes : le peuple français est très souvent présenté chez MLP comme un ensemble de citoyens frustrés et victimes des circonstances et, dans le discours d'EM, le peuple est présenté comme capable, visionnaire et optimiste. Dans le discours de MLP, 40 nominations sur 132 des catégories socio-économiques témoignent de la tendance de la candidate du Front national à présenter les gens comme souffrants et mécontents. Dans le discours d'EM, 58 dénominations sur 158 des catégories socio-économique témoignent de la tendance du candidat du mouvement En Marche à parler des capacités et des possibilités du 
peuple. Ainsi, au total, sur les 220 nominations du peuple prononcées par MLP, 83 sont négativement connotées, et sur les 246 nominations du peuple prononcées par EM, 87 sont positivement connotées.

\section{Citoyens français et identité nationale : éléments de conclusion}

$\mathrm{Au}$ début de cette étude, nous nous sommes posé la question de savoir si les différences de fréquence de types et de contextes dépendent:

a. de l'orientation politique du/de la candidate?

Emmanuel Macron se veut un leader pour toutes et tous. Le choix de la forme «concitoyens » représente parfaitement cette volonté. Dans le domaine économique, il envisage une politique de réformes sur le marché du travail et dans le secteur économique ; il mise ainsi sur la population active. Il associe ces nominations aux réformes et à une volonté politique. Marine Le Pen, avec la nomination «compatriotes », véhicule l'image d'un peuple français homogène qui partagerait les mêmes valeurs, dont la plus importante est l'amour de la patrie.

b) des besoins de renforcement de l'ethos du/de la candidate?

Marine Le Pen cherche à se présenter comme la protectrice de la patrie ; elle exprime, à de nombreuses reprises, sa solidarité avec les plus modestes, qu'elle oppose à la position d'EM, du côté des plus aisés, selon elle. Elle emploie ainsi des mots qu'elle associe à la politique et à l'économie «sauvage » d'Emmanuel Macron, où «les gros mangent les petits ».

De son côté, Emmanuel Macron cherche à donner l'image d'un homme ouvert qui voit les potentialités de la France et qui a de la force pour réaliser des réformes lourdes. Avec l'emploi systématique de «concitoyens » pour parler du peuple français, il inclut tout le monde, dans une communauté construite sur les valeurs universelles et humaines et non sur une vision étroite d'une France repliée sur elle-même.

Enfin, nous nous sommes demandé quelles stratégies électorales pourraient être liées à ces nominations. Emmanuel Macron cherche à rassembler le peuple français, à créer de l'unité. Il utilise plus souvent des dénominations «neutres ». Tout le long du débat, Marine Le Pen cherche à donner au peuple à l'écoute un sentiment d'une élite dominante distante qui ne les écoute pas. Elle cherche aussi à donner l'idée d'une société divisée où les gens ont de moins en moins de pouvoir et de liberté Un grand nombre de nominations du «peuple » sont présentées dans des contextes où les gens sont décrits comme impuissants, victimes et soumis à l'économie de Bruxelles des décisions qui sont prises loin de là où ils sont.

\section{Bibliographie}

Calabrese Steimberg, Laura. 2012. « L'acte de nommer : nouvelles perspectives pour le discours médiatique », Langage et société 2012/2 ( $\left.\mathrm{n}^{\circ} 140\right)$, p. 29-40. DOI $10.3917 / \mathrm{ls} .140 .0029$ 
Charaudeau (2011) «Réflexions pour l'analyse du discours populiste », revue Mots, ${ }^{\circ} 97$, Les collectivités territoriales en quête d'identité, pp.101-116, ENS Éditions, Lyon, 2011 [TEXTE INTEGRAL]

Frath, Pierre. 2015. «Dénomination référentielle, désignation, nomination » In Langue française 2015/4 ( $\left.\mathrm{N}^{\circ} 188\right)$, p. 33-46. DOI 10.3917/lf.188.0033

Kerbrat-Orecchioni, C. 2017. Les débats de l'entre-deux-tours des élections présidentielles françaises. Constances et évolutions d'un genre. Paris : L'Harmattan.

Kleiber, Georges. 2001. «Remarques sur la dénomination» In Cahiers de praxématique 36. 21-41. Montpellier : Pulm.

Kleiber, G. (2001) Remarques sur la dénomination. Cahiers de praxématique 36. Montpellier : Pulm. 21-41

Langue Française 2012/2 (n¹74) La Dénomination https://www.cairn.info/revue-languefrancaise-2012-2.htm

Langue française 2015/4 ( $\mathrm{N}^{\circ}$ 188) Stabilité et instabilité dans la production du sens : la nomination en discours https://www.cairn.info/revue-langue-francaise-2015-4.htm

Longhi, Julien. 2015. «Stabilité et instabilité dans la production du sens : la nomination en discours » In Langue française 2015/4 ( $\left.\mathrm{N}^{\circ} 188\right)$, p. 5-14. DOI 10.3917/lf.188.0005

Moirand, Sophie. 2004. « De la nomination au dialogisme : quelques questionnements autour de l'objet de discours et de la mémoire des mots ", in A. Cassanas et al. (éds), Dialogisme et nomination, Montpellier : Presses universitaires de la Méditerranée, 2761.

Roitman, Malin \& Sullet-Nylander, Françoise. 2010a. «Voix de campagne présidentielle : quelques observations sur la question et la réfutation dans le débat télévisé RoyalSarkozy (2 mai 2007) » In Abecassis M. \& Ledegen, G. (eds). Les voix des français : à travers l'histoire, l'école et la presse. Oxford : Peter Lang.

Siblot, Paul. 2001. «De la dénomination à la nomination. Les dynamiques de la signifiance nominale et le propre du nom » In Cahiers de praxématique 36. 189-214. Montpellier : Pulm.

Sullet-Nylander, Françoise \& Roitman, Malin. 2010b. «De la confrontation politicojournalistique dans les grands duels politiques télévisés : questions et préconstruits » In Burger M., Jacquin, J. et Micheli, R. (eds). Le français parlé des médias : les médias et le politique », Lausanne : Centre de linguistique et des sciences du langage. http://www.unil.ch/clsl/page81503.html

Sullet-Nylander, Françoise \& Roitman, Malin. 2011. «Discours rapportés et débats télévisés : Étude comparative : Chirac/Jospin (1995) vs Sarkozy/Royal (2007) » In Marnette, S., López, M. et Rosiers, L. (Eds). Citations II. Citer pour quoi faire ? Pragmatique de la citation. Louvain-la-Neuve : Academia Bruylant.

Sullet-Nylander, Françoise \& Roitman, Malin. 2016. « Mais vous avez tout à fait raison M. le Premier ministre. Termes d'adresse et débats politiques télévisés de l'entre-deuxtours » In Hernández-Flores, N. \& Bravo, D. (eds). Pragmatica Sociocultural. De Gruyter Mouton. 